\title{
Fortschritte in der Nephrologie: Vorteilhaft für Patienten und Kosten
}

\section{Pierre-Yves Martin}

Nephrologische Abteilung HUG, Präsident der Schweizerischen Gesellschaft für Nephrologie

\author{
Das seltenere Fortschreiten von Nierenerkrankungen hin zur terminalen Nierenin- \\ suffizienz ist ein wenig sichtbarer, aber klar vorhandener Nutzen für die Patienten, \\ der langfristig auch Kostenvorteile bringt. Jeder Patient, bei dem ein Fortschreiten \\ bis zur Dialyse abgewendet werden kann, geniesst eine höhere Lebensqualität - \\ und spart Kosten von rund 250000 Franken.
}

Die seit den siebziger Jahren in der Schweiz angebotenen Möglichkeiten zur Dialyse und Nierentransplantation ermöglichen tausenden Menschen ein Leben trotz unzureichender Nierenfunktion. Seit den siebziger Jahren ist der Einsatz dieser Nierenersatzverfahren ständig gestiegen, so dass aktuell mehr als 10000 Menschen in der Schweiz dank dieser Behandlungen am Leben sind [1]. Die gewonnene Überlebenszeit ist oft beträchtlich: 55\% der Personen zwischen 18 und 64 Jahren, die im Jahr 2004 auf ein solches Verfahren angewiesen waren, lebten 10 Jahre später noch.

Frau $\mathrm{X}$ leidet unter einer angeborenen Nierenerkrankung, die sich im Alter von 30 Jahren in Richtung einer terminalen Insuffizienz entwickelt. Sie beginnt eine ambulante Peritonealdialyse, die es ihr ermöglicht, in Vollzeit als Wirtschaftsprüferin weiterzuarbeiten. Zudem wird sie auf die Warteliste für eine Nierentransplantation gesetzt. Ihr Ehemann möchte ihr eine Niere spenden, weist aber eine andere Blutgruppe auf. Nach zwei Jahren Peritonealdialyse ist die Patientin zunehmend müde und kann nur noch unter Mühen weiterarbeiten. Das Paar entscheidet sich für eine seit einigen Jahren in der Schweiz mögliche ABO-inkompatible Transplantation (da sie ja unterschiedliche Blutgruppen aufweisen). Die Transplantation verläuft erfolgreich, und Frau $X$ arbeitet sechs Monate nach dem Eingriff wieder Vollzeit. Zwei Jahre danach bekommen die beiden ihr erstes Kind.

Durch die Einführung der ambulanten Peritonealdialyse und zuletzt der Heimhämodialyse steht inzwischen eine grössere Auswahl an verbesserten Dialysetechniken zur Verfügung, was die Lebensqualität der Patienten deutlich verbessert. Auch bei Nierentransplantationen, die bei terminaler Niereninsuffizienz die beste Option darstellen, hat der medizinische Fortschritt dazu geführt, dass solche Transplantationen für mehr Betroffene möglich sind und vom Körper besser angenommen werden. Bei der Lebendspende sind grosse Fortschritte zu verzeichnen. Inzwischen sind $\mathrm{ABO}$-inkompatible Nierentransplantationen möglich, und ein Programm für Überkreuzspenden erhöht die Chance, einen passenden Spender zu finden, deutlich. Allerdings gibt es Einschränkungen. Die erste Einschränkung ist medizinischer Art: Mehr als 50\% der Patienten, bei denen es zur terminalen Niereninsuffizienz kommt, können - beispielsweise aufgrund kardiovaskulärer Risikofaktoren oder ihres hohen Alters - eine solche Behandlung nicht in Anspruch nehmen. Die zweite Einschränkung ergibt sich aus der

Therapien, die ein Fortschreiten in Richtung terminaler Niereninsuffizienz verhindern können, sind das beste Mittel, um die finanziellen Kosten von Niereninsuffizienzen zu verringern.

niedrigen Quote postmortaler Spenden in der Schweiz, die durch die hohe Quote an Lebendspenden, meist vom Partner oder einem anderen Familienmitglied, nur zum Teil ausgeglichen werden kann. Dies führt dazu, dass immer mehr Menschen auf der Warteliste stehen und die mittlere Wartezeit sehr lang ist (3 bis 4 Jahre). Es hat aber auch finanzielle Folgen: Schliesslich ist die Nierentransplantation die kostengünstigste Form der Behandlung einer terminalen Niereninsuffizienz.

Dennoch nehmen auch diese Behandlungen das Gesundheitssystem sehr stark in Anspruch, da Dialyse und Nierentransplantation teure Infrastrukturen erfordern. Da die Anzahl der Patienten und ihre Lebenserwartung steigen, erhöhen sich auch Jahr für Jahr die 
Kosten. Aus diesem Grund steht die Behandlung der terminalen Niereninsuffizienz im Visier der Entscheidungsträger des Gesundheitswesens und von Sparforderungen. Dies führt zu enormen Spannungen zwischen den Akteuren in der Nephrologie und den Leistungsfinanzierern, da sich der Grossteil der in diesen Strukturen entstehenden Kosten kaum verringern lässt.

\section{Die Herausforderung: bei mehr Patienten die terminale Niereninsuffizienz ver- meiden}

Der beste Weg, um die Lage der Patienten zu verbessern und zugleich die Behandlungskosten zu senken, ist, es bei möglichst wenigen Patienten zur terminalen Niereninsuffizienz (TNI) kommen zu lassen. Da die meisten TNI-Patienten über 75 Jahre alt sind, erscheint dies bei einer alternden Bevölkerung schwierig. Andererseits nehmen die beiden häufigsten Ursachen für eine terminale Niereninsuffizienz - Typ-II-Diabetes und arterielle Hypertonie - immer weiter zu [2].

HerrY, 65 Jahre alt, Raucher, leidet seit 20 Jahren unterTyp-II-Diabetes und Bluthochdruck; begleitet von einer Gewichtszunahme um mehr als $20 \mathrm{~kg}$. Aufgrund seiner zahlreichen beruflichen und sozialen Aktivitäten konnte er nie eine Diät verfolgen; sein Diabetes und sein Bluthochdruck werden nur unzureichend behandelt. Als er eine progressive Niereninsuffizienz entwickelt, sagt man inm eine Dialysepflichtigkeit innerhalb von drei Jahren voraus. Nach seiner Pensionierung hat er nun seine Ernährung umgestellt und nimmt weniger Salz und Proteine, aber mehr Gemüse zu sich. Er raucht nicht mehr und ist körperlich aktiver ( $3 \times 1$ Stunde Gehen/Woche). Dank einer medikamentösen Behandlung haben sich seine Blutdruckwerte verbessert, ebenso die Stoffwechselazidose, die mit seiner Niereninsuffizienz einhergeht. Mit 67 Jahren hat er nun $13 \mathrm{~kg}$ abgenommen, der Diabetes ist besser kontrolliert und die Niereninsuffizienz hat sich stabilisiert. Falls Herr $Y$ in seinem Leben noch Dialyse-Patient wird, dürfte dies frühestens 15 Jahre später eintreten.

Indessen erleben wir seit zwei Jahrzehnten grosse Fortschritte im Verständnis der Pathogenese chronischer Niereninsuffizienz. Chronische Nierenerkrankungen können in unterschiedlichen Kontexten auftreten: Sie können die Folge einer akuten Nierenschädigung (akute Niereninsuffizienz unterschiedlichster Ursache) sein, die irreversibel wird. Sie können auch durch systemische Autoimmun- oder Stoffwechselerkrankungen ausgelöst werden, wenn sich bei deren Behandlung Nierenschädigungen nicht vermeiden lassen. Beide Situationen führen zum Verlust von Nephronen, den strukturellen und funktionalen Untereinheiten einer Niere. Dieser Verlust wiederum bewirkt eine Überlastung der noch funktionsfähigen Nephrone, was ihre Alterung beschleunigt und eine progressive Degeneration zur Folge hat. Dadurch wird der Nephronenverlust noch einmal verschlimmert. Der Prozess setzt sich bis zum totalen Funktionsverlust fort.

Das Verständnis dieses Phänomens hat zum Konzept der «Nephroprotektion» geführt, das eine Vielzahl an Massnahmen im Bereich der Medikation, der Ernährung und des Lebensstils umfasst. Eine chronische Niereninsuffizienz lässt sich immer noch nicht heilen, ihr Fortschreiten aber zumindest verlangsamen, wenn nicht gar stoppen. Dazu gibt es eine Reihe von Instrumenten, die wir kombinieren können. Spezielle Medikamente (ACE-Hemmer/Sartane) sind unverzichtbar,

\section{Die Kosten erhöhen sich Jahr für Jahr - da die Anzahl der Patienten und ihre Lebens- erwartung steigen.}

und ihr Nutzen ist gut belegt [3]. Laut sämtlichen veröffentlichten Studien bremsen diese Therapien die Progression der Niereninsuffizienz, wenn auch mit unterschiedlichem Erfolg. Auch der Verzicht auf Tabak, die Senkung der Säurebelastung durch eine Ernährung, die ärmer an Proteinen und stärker an Basen ist, wirken sich positiv aus. Der frühzeitige Einsatz von Statinen sorgt für kardiovaskulären Schutz. Das Aufkommen von SGLT2-Hemmern in der Behandlung von Typ-II-Diabetes erweist sich in der diabetischen Nephropathie als vielversprechend, und die ersten Studien zeigen, dass diese das Fortschreiten der diabetischen Nephropathie bremsen [4].

\section{Verlangsamtes Fortschreiten der Nieren- insuffizienz dank medikamentöser Therapien}

Die Beine von Herrn Z., 40 Jahre alt, schwellen innerhalb weniger Tage enorm an. Es wird eine membranöse Glomerulonephritis in Verbindung mit Antikörpern gegen PLA2R diagnostiziert; diese Autoimmunerkrankung stellt die Hauptursache für primäre membranöse Glomerulonephritis dar. Unbehandelt beträgt das Risiko, dass sich eine terminale Niereninsuffizienz entwickelt, $50 \%$. Die verfügbaren Therapien beinhalten hohe Dosen an Kortison und Endoxan. Eine solche immunsuppressive Therapie geht mit zahlreichen Nebenwirkungen einher. Die Ärzte entscheiden sich für Rituximab. Zwar ist diese Behandlung noch nicht für diese Indikation registriert; zahlreiche Studien belegen aber ihre Wirksamkeit und ihr besseres Profil an Nebenwirkungen. Sechs Monate nach der Behandlung lassen die Symptome nach. Auch nach fünf Jahren ist kein Rückfall festzustellen, die Nierenfunktion ist normal.

Eine der Ursachen für Niereninsuffizienzen ist Glomerulonephritis. Bei einem grossen Teil der hiervon betroffenen Patienten ist ein Fortschreiten in Richtung terminaler Niereninsuffizienz (TNI) zu verzeichnen, 
sodass diese Erkrankung letztlich eine der wichtigsten Ursachen für TNI darstellt. Biologische Therapien wie Rituximab oder Eculizumab, die eigentlich für nicht nephrologische Indikationen entwickelt wurden, erweisen sich bei der Behandlung zahlreicher Glomerulonephritiden als sehr wirksam [5]. Zwar werden diese Therapien für solche Indikationen, die zumeist noch nicht registriert sind, aufgrund ihres Preises bislang nur wenig eingesetzt, sie dürften aber zu einer Verbesserung der renalen Prognose von Glomerulonephritiden führen. Dies lässt sich bereits in der Schweiz und in den USA feststellen.

\section{Eine chronische Niereninsuffizienz lässt sich immer noch nicht heilen, ihr Fortschreiten aber zumindest verlangsamen, wenn nicht gar stoppen.}

Ebenso gibt es Hoffnung bei der Behandlung der polyzystischen Nierendegeneration. Hierbei handelt es sich um eine Erbkrankheit, die zur Bildung zahlreicher Zysten in den Nieren führt. Seit einem Jahr ist ein spezielles Medikament, Tolvaptan, verfügbar [6]. Dieses Medikament bremst das Zystenwachstum und verlangsamt dadurch das Fortschreiten der Niereninsuffizienz mit einer Wirksamkeit, die in den ersten Jahren derjenigen von ACE-Hemmern entspricht. Angesichts der Tatsache, dass sich bei $50 \%$ der Menschen, die unter dieser Erbkrankheit leiden, eine terminale Niereninsuffizienz entwickelt und diese mehr als 10\% der Patienten ausmachen, dürften in einigen Jahren, falls sich eine langfristige Wirksamkeit dieses Medikaments bestätigt, entsprechende Erfolge zu verzeichnen sein.

\section{Neben ihrem Nutzen für die Patienten bieten erfolgreiche Therapien auch wirtschaftliche Vorteile}

Therapien, die ein Fortschreiten in Richtung terminaler Niereninsuffizienz verhindern können, bieten

Korrespondenz: Pierre-Yves Martin, M.D. Head of Nephrology Service Director of the Department of Medical Specialties University Hospital of Geneva 4 rue Gabrielle-Perret-Gentil CH-1211 Genève 14 Tél.: +4122 3729761 Geneva, Switzerland President of the Swiss-Society of Nephrology 2016-2017 lyse beginnen. Wenn diese Zahl um 10\% gesenkt werden könnte, liessen sich im Schweizer Gesundheitswesen rund 21 Millionen Franken einsparen. Die seit kurzem erhältlichen und die in Entwicklung befindlichen Medikamente bieten auf jeden Fall ein solches Potenzial.

\section{Zusammenfassung}

Seit den siebziger Jahren haben sich die Dialyse- und Transplantationstechniken eindrücklich weiterentwickelt und diversifiziert. Die dadurch zunehmenden und besseren Behandlungsmöglichkeiten haben die Lebenszeit und die Lebensqualität der Patienten deutlich erhöht. Dank dieser seither ständig zunehmenden Behandlungen sind aktuell mehr als 10000 Menschen in der Schweiz am Leben. Allerdings beanspruchen diese Behandlungen erhebliche Ressourcen unseres Gesundheitssystems, da sie teure Infrastrukturen erfordern und hohe Kosten verursachen. Therapien, die ein Fortschreiten in Richtung terminaler Niereninsuffizienz verhindern können, bieten nicht nur den Patienten grossen Nutzen, sondern sind auch das beste Mittel, um die finanziellen Kosten von Niereninsuffizienzen zu verringern. Pro Patient, bei dem eine Dialyse verhindert werden kann, können Kosten in Höhe von 250000 Franken eingespart werden. Angesichts der Zahl betroffener Patienten bieten die seit kurzem erhältlichen und die in Entwicklung befindlichen Medikamente ein grosses Potenzial zur Behandlung - und zur Kostensenkung.

Literatur

1 Pippias M, Kramer A, Noordzij M, et al. The European Renal Association - European Dialysis and Transplant Association Registry Annual Report 2014: a summary. Clin Kidney J.2017 Apr;10(2):154-69.

2 Heaf J. Current trends in European renal epidemiology. Clin Kidney J.2017 Apr;10(2):149-53.

3 Ruiz-Hurtado G, Sarafidis P, Fernández-Alfonso MS, et al. Global cardiovascular protection in chronic kidney disease. Nat Rev Cardiol. 20160ct;13(10):603-8.

4 Wanner C, Inzucchi SE, Lachin JM,et al. EMPA-REG OUTCOME Investigators. Empagliflozin and Progression of Kidney Disease in Type 2 Diabetes. N Engl J Med.2016 Jul 28;375(4):323-34.

5 Holdsworth SR, Gan PY, Kitching AR. Biologics for the treatment of autoimmune renal diseases. Nat Rev Nephrol. 2016 Apr;12(4):217-31.

6 Gansevoort RT, Arici M, Benzing T,et al. Recommendations for the use of tolvaptan in autosomal dominant polycystic kidney disease a position statement on behalf of the ERA-EDTA Working Groups on Inherited Kidney Disorders and European Renal Best Practice. Nephrol Dial Transplant. 2016 Mar;31(3):337-48.

7 Lederer ED. America's kidney disease epidemic merits congressional attention. The Hill. Thehill.com/blogs/congress-blog/ healthcare/ 